See discussions, stats, and author profiles for this publication at: https://www.researchgate.net/publication/308274424

\title{
Relationships of feedlot performance, feeding behavior, rumen morphometrics, and carcass characteristics of Nellore cattle differing in phenotypic residual feed intake
}

Article in Journal of Animal Science · October 2016

DOI: 10.2527/jas.2016-0579

CITATION

1

9 authors, including:

Mário De Beni Arrigoni

São Paulo State University

119 PUBLICATIONS 693 CITATIONS

SEE PROFILE

Some of the authors of this publication are also working on these related projects:

Rumenology Book View project
READS

113

Danilo Millen

São Paulo State University (UNESP)

77 PUBLICATIONS 331 CITATIONS

SEE PROFILE 


\title{
Relationships of feedlot performance, feeding behavior, rumen morphometrics, and carcass characteristics of Nellore cattle differing in phenotypic residual feed intake ${ }^{1}$
}

\author{
M. C. S. Pereira, * G. D. Cruz, $\dagger$ M. D. B. Arrigoni, * A. L. N. Rigueiro,* \\ J. Silva, \$ T. V. B. Carrara,* P. C. S. Santos, + L. L. Cursino, + and D. D. Millen ${ }^{2}$ \\ *Department of Breeding and Animal Nutrition, São Paulo State University, \\ Botucatu, São Paulo, Brazil 18618-000; †Purina Animal Nutrition LLC, Shoreview, Minnesota 55126; \\ and \$College of Technology and Agricultural Sciences, São Paulo State University, Dracena, São Paulo, Brazil 17900-000
}

\begin{abstract}
The objective of this study was to examine the relationship of DMI fluctuation, feedlot performance, feeding behavior, rumen morphometrics, and carcass characteristics in Nellore cattle classified by residual feed intake (RFI). One experiment was conducted in 2 consecutive years using individual pens $(1.0 \times 7.0 \mathrm{~m})$ at the São Paulo State University feedlot, Dracena campus, Brazil. The experiment in year 1 started in June of 2012 with forty-eight 20-mo-old Nellore yearling bulls with an initial BW of $358.2 \pm$ $19.4 \mathrm{~kg}$. The experiment in year 2 started in January of 2013 with sixty 20-mo-old Nellore yearling bulls with an initial BW of $402.5 \pm 33.0 \mathrm{~kg}$. Experiments in years 1 and 2 lasted 94 and 84 d, respectively. All yearling bulls were categorized as high RFI $(>0.5$ SD above the mean, $n=25)$, medium RFI $( \pm 0.5 \mathrm{SD}$ from the mean, $n=56)$, and low RFI $(<0.5 \mathrm{SD}$ below the mean, $n=27)$. Visual appraisal to collect behavior data was made on $\mathrm{d} 40$ (finishing period) of both years. Yearling bulls were harvested when average across treatment
\end{abstract}

groups achieved a fat thickness of $4 \mathrm{~mm}$ at the 12th rib. Low-RFI yearling bulls had lower daily DMI, expressed either in kilograms $(P<0.01)$ or as percentage of BW $(P<0.01)$, and improved G:F $(P<0.01)$ when compared to high-RFI animals. No differences were observed $(P>0.10)$ for ADG, final BW, or $\mathrm{HCW}$ among RFI groups. Also, low-RFI yearling bulls had thinner final 12th rib $(P<0.01)$ and biceps femoris (P8) fat thickness $(P<0.01)$. Low-RFI yearling bulls were slower to consume $(P=0.03)$ and ruminate $(P<$ $0.01) 1 \mathrm{~kg}$ of either DM or NDF. No significant $(P>$ $0.10)$ RFI effect was observed for any ruminal morphometrics variables evaluated, with the exception of papillae area, in which low-RFI Nellore yearling bulls tended to have smaller $(P=0.07)$ papillae area than medium-RFI animals. In general, low-RFI Nellore yearling bulls consumed more particles larger than 19 and $8 \mathrm{~mm}$ and had a similar performance when compared to both medium- and high-RFI bulls; however, carcass fat composition was negatively impacted.

Key words: carcass, fat, feedlot, Nellore, performance, residual feed intake

(C) 2016 American Society of Animal Science. All rights reserved. J. Anim. Sci. 2016.94:4287-4296 doi:10.2527/jas2016-0579

\section{INTRODUCTION}

Flint corn is the primary grain source used in finishing diets in Brazil, and most of the feedlots use cracking as the most common (57.6\%) processing method (Oliveira and Millen, 2014). As a result, when compared to steam flaking, which is commonly used in U.S.

\footnotetext{
${ }^{1}$ Financial support was provided by FAPESP (São Paulo Research Foundation, São Paulo, São Paulo, Brazil).

${ }^{2}$ Corresponding author: danilomillen@dracena.unesp.br

Received April 26, 2016.

Accepted July 11, 2016.
}

feedlot diets, Brazilian diets are intrinsically less efficient when used by the animal (Simas et al., 2008).

In addition, according to NRC (2000), about $80 \%$ of total dietary energy consumed by beef cattle is used to maintain normal body functions, and it has been estimated that Bos indicus maintenance requirements are $10 \%$ less than Bos taurus, which normally reduces DMI (Pacheco et al., 2012). Thus, improvements in feed efficiency will directly lead to cost reduction and better overall production system efficiency. Herd et al. (2004) and Cruz et al. (2010) reported associations of residual feed intake (RFI) with biological processes affecting economically important traits in Bos taurus 
cattle, such as DMI and G:F. Moreover, Nascimento et al. (2015) reported the same associations for Nellore (Bos indicus), which differs from a Bos taurus breed in terms of DMI, body composition, and feeding behavior (Turner, 1980; Schutt et al., 2009). Animals classified as low RFI are more efficient than high-RFI animals because they consume less feed for similar gain.

In the last decade significant progress has been made to identify physiological mechanisms related to RFI, and the main contributors were tissue metabolism, heat increment, feeding behavior and activity, and feed digestibility (Richardson and Herd, 2004; Nkrumah et al., 2006; Cruz et al., 2010; Lancaster et al., 2009). Yet differences in DMI fluctuation, selective consumption of ration particles, and rumen morphometric have not been investigated in the RFI literature.

Thus, the objective of this study was to examine the effects of RFI grouping on feedlot performance and carcass traits variables, DMI fluctuation, feeding behavior, selective consumption, and rumen morphometrics of Nellore cattle.

\section{MATERIALS AND METHODS}

All the procedures involving the use of animals in this study were in accordance with the guidelines established by the São Paulo State University Ethical Committee for Animal Research (Ministério da Ciência, Tecnologia e Inovação Conselho Nacional de Controle de Experimentação Animal, 2013).

\section{Animals, Nutritional Management, and Performance Measurements}

One study was conducted and replicated in 2 consecutive years using individual pens $(1.0 \times 7.0 \mathrm{~m})$ at the São Paulo State University feedlot, Dracena campus, Brazil. In both years, animals were provided by the same commercial farm and transported for about $40 \mathrm{~km}$ to the university's feedlot. In the first year, the study started in June of 2012, with forty-eight 20-mo-old Nellore yearling bulls with an initial BW of $358.2 \pm 19.4 \mathrm{~kg}$ and lasted $94 \mathrm{~d}$. In the second year, the study started in January of 2013, with sixty 20-mo-old Nellore yearling bulls with an initial BW of $402.5 \pm 33.0 \mathrm{~kg}$ and lasted $84 \mathrm{~d}$.

At the beginning of the study in each year, all yearling bulls were dewormed and vaccinated (tetanus, bovine viral diarrhea virus, 7-way Clostridium sp.; Cattlemaster and Bovishield, Pfizer Animal Health, New York, NY). Yearling bulls were fed ad libitum twice daily at $0800 \mathrm{~h}$ ( $40 \%$ of total ration) and $1500 \mathrm{~h}$ (60\% of total ration), targeting $3 \%$ to $5 \%$ refusal. The experimental diets were formulated according to the Large Ruminant Nutrition System (Fox et al., 2004) and are shown in Table 1. The adaptation program consisted of 2 adaptation diets, which contained $68 \%$ and $76 \%$ concentrate and were fed ad libitum over a period of $7 \mathrm{~d}$ each. Experimental diets were composed of sugarcane bagasse, cracked corn grain, soybean meal, and a supplement containing urea (Table 1).

The amount of feed offered was adjusted every day on the basis of the amount of feed refusals before each morning delivery. Feed offered and refused was weighed daily in the morning and sampled weekly for chemical analysis. The DMI was calculated daily and expressed in kilograms and as a percentage of BW. Yearling bulls had free-choice water access to a water trough $(0.89 \times 1.00 \times 1.00 \mathrm{~m})$. Cattle were withheld from feed for $16 \mathrm{~h}$ before every BW assessment, which was performed every 4 wk in both years.

Final BW was obtained at feedlot prior to harvest. At the commercial abattoir, a captive bolt device was used for stunning cattle prior to slaughter. The HCW was obtained after KPH fat removal. Dressing percentage was calculated by dividing $\mathrm{HCW}$ by final BW. Biceps femoris fat thickness (P8) and 12th rib fat thickness were measured via ultrasound at the beginning and at the end of the study in both years, following the method described by Perkins et al. (1992). Images were collected using an Aloka SSD1100 Flexus RTU unit (Aloka Co. Ltd., Tokyo, Japan) with a $17.2-\mathrm{cm}, 3.5-\mathrm{MHz}$ probe. Rib fat thickness was measured at the 12th to 13th rib interface over the LM, two-thirds the distance from the spine between the medial and lateral muscle ends. A single trained technician scanned and analyzed all images. Yearling bulls were harvested when average across treatment groups achieved 4-mm fat thickness at the 12th rib. Cattle were transported $150 \mathrm{~km}(\sim 3 \mathrm{~h})$ to a commercial abattoir.

\section{Measures of Feed Efficiency}

Average daily gain during the test period for both years was calculated by fitting a linear regression through all BW observations of each bull. Similarly, midtest metabolic $\mathrm{BW}\left(\mathrm{BW}^{0.75}\right)$ was estimated from the intercept and slope of the regression line after fitting a linear regression through all metabolic BW observations. The G:F was calculated by dividing the overall ADG by DMI. The RFI was calculated as the residual of the regression equation of observed DMI as a function of ADG and average BW $\mathrm{W}^{0.75}$ (Archer et al., 1997):

$$
\mathrm{DMI}=\beta_{0}+\beta_{1} \times \mathrm{BW}^{0.75}+\beta_{2} \times \mathrm{ADG}+\varepsilon,
$$

where $\beta_{0}$ is the $y$ intercept, $\beta_{1}$ is the partial regression coefficient of midtest $\mathrm{BW}^{0.75}, \beta_{2}$ is the partial regression coefficient of $\mathrm{ADG}$, and $\varepsilon$ is the error term. 
Residual BW gain (RG) was calculated as the residual of the regression equation of observed ADG as a function of DMI and average BW ${ }^{0.75}$ (Crowley et al., 2010]):

$$
\mathrm{ADG}=\beta_{0}+\beta_{1} \times \mathrm{BW}^{0.75}+\beta_{2} \times \mathrm{DMI}+\varepsilon,
$$

where $\beta_{0}$ is the $y$ intercept, $\beta_{1}$ is the partial regression coefficient of midtest $\mathrm{BW}^{0.75}, \beta_{2}$ is the partial regression coefficient of DMI, and $\varepsilon$ is the error term.

Residual intake - weight gain (RIWG) is a linear function of both RFI and RG and was calculated according to Berry and Crowley (2012):

$$
-1 \times \mathrm{RFI}+\mathrm{RG} \text {. }
$$

Multiplying RFI by -1 accounted for a negative RFI being favorable compared with a positive $R G$ being favorable.

The Kleiber ratio was obtained by dividing ADG by average $\mathrm{BW}^{0.75}$ (Kleiber, 1947).

In summary, positive values for RG, RIWG, and the Kleiber ratio and a negative value for RFI represent efficient animals.

\section{DMI Fluctuations}

Daily DMI fluctuation was calculated for each animal as the difference in intake between consecutive days throughout the study (Bevans et al., 2005). Daily DMI fluctuation was expressed in kilograms and as a percentage of fluctuation according to

$$
\begin{aligned}
& \mathrm{DMI} \text { fluctuation, \% } \%\left[\left(\mathrm{DMI}_{\text {current day }}(\mathrm{kg})-\right.\right. \\
& \left.\left.\mathrm{DMI}_{\text {previous day }}(\mathrm{kg})\right) / \mathrm{DMI}_{\text {previous day }}(\mathrm{kg})\right] \times 100 .
\end{aligned}
$$

\section{Feeding Behavior and Selective Consumption}

Data collection of behavior was made visually on d 40 (finishing period) of both years of the study using a method adapted from Robles et al. (2007). Feeding behavior data were recorded by 24 trained individuals (1 per pen) every 5 min during a 24-h period for each animal as follows: time spent eating, ruminating, resting (expressed in minutes), and number of meals per day. A meal was considered the noninterrupted time cattle stayed in the feed bunk eating the ration.

The DMI were measured on the days of data collection. The meal length in minutes was calculated by dividing time spent eating by number of meals per day. The DMI per meal in kilograms was calculated by dividing DMI by the number of meals per day. Also, time spent eating and time spent ruminating data

\begin{tabular}{|c|c|c|c|}
\hline \multirow[b]{2}{*}{ Item } & \multicolumn{3}{|c|}{ Percentage of concentrate } \\
\hline & $68 \%$ & $76 \%$ & $84 \%$ \\
\hline Period & Adaptation 1 & Adaptation 2 & Finishing \\
\hline \multicolumn{4}{|l|}{ Ingredients, $\% \mathrm{DM}$} \\
\hline Sugarcane bagasse & 32.0 & 24.0 & 16.0 \\
\hline Corn grain, cracked & 48.1 & 58.0 & 71.5 \\
\hline Soybean meal & 16.7 & 14.05 & 7.70 \\
\hline Supplement ${ }^{1}$ & 3.20 & 3.95 & 4.80 \\
\hline \multicolumn{4}{|l|}{ Nutrient content, ${ }^{2} \% \mathrm{DM}$} \\
\hline DM, \% & 71.0 & 75.0 & 79.0 \\
\hline TDN & 71.0 & 74.0 & 76.0 \\
\hline $\mathrm{NE}_{\mathrm{g}},{ }^{3} \mathrm{Mcal} / \mathrm{kg}$ & 1.06 & 1.12 & 1.18 \\
\hline Total NFC ${ }^{3,4}$ & 46.0 & 51.0 & 57.0 \\
\hline $\mathrm{CP}^{5}$ & 15.5 & 15.5 & 14.2 \\
\hline Ether extract ${ }^{5}$ & 3.6 & 3.8 & 3.9 \\
\hline $\mathrm{NDF}$ & 33.0 & 27.9 & 22.8 \\
\hline $\mathrm{Ca}^{5}$ & 0.49 & 0.55 & 0.62 \\
\hline $\mathrm{P}^{5}$ & 0.34 & 0.35 & 0.34 \\
\hline
\end{tabular}

Table 1. Feed ingredients and chemical composition of the experimental diets fed to Nellore yearling bulls consuming high-concentrate diets $(n=108)$

${ }^{1}$ Supplement contained $26.66 \%$ of urea as a $\mathrm{N}$ source, as well as $\mathrm{Ca}$, $11.31 \%$; P, $1.17 \%$; S, 2,24\%; Mg, 0.88\%; Na, 3.80\%; Co, $0.001 \%$; $\mathrm{Cu}$, $0.02 \%$; I, 0.001\%; Mn, 0.07\%; Se, 0.0005\%; Zn, 0.10\%; vitamin A, 56,430 IU; vitamin D, 7,054 IU; vitamin E, 257 IU; sodium monensin $575 \mathrm{mg}$.

${ }^{2}$ All values except DM (\%) are expressed on a DM basis.

${ }^{3}$ Estimated by equations according to $=$ Large Ruminant Nutrition System (Fox et al., 2004).

${ }^{4}$ Nonfibrous carbohydrates.

${ }^{5}$ Ether extract was determined gravimetrically after extraction using petroleum ether in a Soxhlet extractor (method 920.85; AOAC, 1990). Crude protein, $\mathrm{Ca}$, and $\mathrm{P}$ were determined according to methods 990.02 (AOAC, 1997), 968.08 (AOAC, 1995), and 965.17 (AOAC, 1990), respectively.

were used to calculate the eating rate of DM (ERDM; time spent eating/DMI) and rumination rate of DM (RRDM; time spent ruminating/DMI), both expressed in minutes per kilogram of DM. Samples of diets and orts were collected for chemical analysis of NDF (Van Soest et al., 1991) to determine the intake of NDF on the day of feeding behavior data collection. Eating rate of NDF (ERNDF) was calculated by dividing the time spent eating by NDF intake. Rumination rate of NDF (RRNDF) was determined by dividing the time spent ruminating by NDF intake. Both ERNDF and RRNDF were expressed in minute per kilogram of NDF.

Samples of diets and orts were also collected on $d$ 40 of both years of the study for determination of particle-size distribution, which was performed by sieving using the Penn State Particle Size Separator and reported on an as-fed basis as described by Heinrichs and Kononoff (1996). Selective consumption was determined as follows: $n$ intake $/ n$ predicted intake, in which $n=$ particle fraction screens of $19 \mathrm{~mm}$ (long), $8 \mathrm{~mm}$ (medium), $1.18 \mathrm{~mm}$ (short), and a pan (fine). Selective consumption values equal to 1 indicate no 
Table 2. Pearson correlations and $P$-values among performance, feeding behavior, and nutrient digestibility variables of Nellore yearling bulls differing in phenotypic residual feed intake ${ }^{1}$

\begin{tabular}{|c|c|c|c|c|c|c|}
\hline Item & Daily DMI, kg & DMI Variation, $\%$ & $\mathrm{ADG}$ & G:F & ERNDF $^{2}$ & RRNDF $^{3}$ \\
\hline$\overline{\mathrm{RFI}}$ & $\begin{array}{c}0.44 \\
(<0.01)\end{array}$ & $\begin{array}{c}-0.18 \\
(<0.01)\end{array}$ & $\begin{array}{c}0.03 \\
(0.80)\end{array}$ & $\begin{array}{c}-0.51 \\
(<0.01)\end{array}$ & $\begin{array}{l}-0.23 \\
(<0.01)\end{array}$ & $\begin{array}{c}-0.10 \\
(0.32)\end{array}$ \\
\hline Daily DMI, kg & & $\begin{array}{l}-0.44 \\
(<0.01)\end{array}$ & $\begin{array}{c}0.74 \\
(<0.01)\end{array}$ & $\begin{array}{c}0.05 \\
(0.61)\end{array}$ & $\begin{array}{l}-0.40 \\
(<0.01)\end{array}$ & $\begin{array}{l}-0.54 \\
(<0.01)\end{array}$ \\
\hline DMI variation, $\%$ & $\begin{array}{l}-0.44 \\
(<0.01)\end{array}$ & & $\begin{array}{l}-0.29 \\
(<0.01)\end{array}$ & $\begin{array}{c}0.04 \\
(0.66)\end{array}$ & $\begin{array}{c}0.12 \\
(0.21)\end{array}$ & $\begin{array}{c}0.18 \\
(0.06)\end{array}$ \\
\hline $\mathrm{ADG}, \mathrm{kg}$ & $\begin{array}{c}0.74 \\
(<0.01)\end{array}$ & $\begin{array}{l}-0.29 \\
(<0.01)\end{array}$ & & $\begin{array}{c}0.69 \\
(<0.01)\end{array}$ & $\begin{array}{c}-0.18 \\
(0.06)\end{array}$ & $\begin{array}{l}-0.36 \\
(<0.01)\end{array}$ \\
\hline G:F & $\begin{array}{c}-0.10 \\
(0.28)\end{array}$ & $\begin{array}{c}-0.02 \\
(0.83)\end{array}$ & $\begin{array}{l}-0.71 \\
(<0.01)\end{array}$ & & $\begin{array}{l}-0.15 \\
(0.12)\end{array}$ & $\begin{array}{c}0.04 \\
(0.65)\end{array}$ \\
\hline ERNDF $^{2}$ & $\begin{array}{c}-0.41 \\
(<0.01)\end{array}$ & $\begin{array}{c}0.12 \\
(0.21)\end{array}$ & $\begin{array}{c}-0.18 \\
(0.06)\end{array}$ & $\begin{array}{l}-0.15 \\
(0.21)\end{array}$ & & $\begin{array}{c}0.46 \\
(<0.01)\end{array}$ \\
\hline RRNDF $^{3}$ & $\begin{array}{c}-0.55 \\
(<0.01)\end{array}$ & $\begin{array}{c}0.18 \\
(0.06)\end{array}$ & $\begin{array}{l}-0.36 \\
(<0.01)\end{array}$ & $\begin{array}{c}0.04 \\
(0.65)\end{array}$ & $\begin{array}{c}0.46 \\
(<0.01)\end{array}$ & \\
\hline
\end{tabular}

${ }^{1} P$-values are in parentheses.

${ }^{2} \mathrm{ERNDF}=$ eating rate of NDF.

${ }^{3} \mathrm{RRNDF}=$ rumination rate of $\mathrm{NDF}$.

sorting, those $<1$ indicate selective refusals (sorting against), and those $>1$ indicate preferential consumption (sorting for).

\section{Rumenitis and Ruminal Morphometrics}

Rumenitis evaluation was performed after cattle evisceration, and all entire washed rumens were scored. Rumen epithelium was classified according to the incidence of lesions (rumenitis) and abnormalities (e.g., papillae clumped) as described by Bigham and McManus (1975) using a scale of 0 (no lesions and abnormalities noted) to 10 (severe ulcerative lesions). All rumens were scored by 2 trained individuals, who were blinded to the treatments, and final data represent the average of the 2 scores.

Also, a $1-\mathrm{cm}^{2}$ fragment of each rumen was collected from cranial sac (atrium ruminis) and placed into a PBS solution for future morphometric measurements according to Resende Júnior et al. (2006). Manually, the number of papillae per square centimeter of rumen wall (NOP) was determined; 12 papillae were randomly collected from each fragment and scanned, and mean papillae area (MPA) was determined using an image analysis system (Image Tool, version 2.01 alpha 4, UTHSCSA Dental Diagnostic Science, San Antonio, TX). The rumen wall absorptive surface area (ASA) in square centimeters was calculated as follows: $1+$ $(\mathrm{NOP} \times \mathrm{MPA})-(\mathrm{NOP} \times 0.002)$, where 1 represents the $1 \mathrm{~cm}^{2}$ fragment collected and 0.002 is the estimated basal area of papillae in square centimeters.

Likewise, a $1-\mathrm{cm}^{2}$ fragment of each rumen was collected from the ventral sac for histological assessment. Histological sections were stained with hematoxylin and eosin, embedded in paraffin wax, and sectioned (Odongo et al., 2006). Histological measurements, such as papillae height, papillae width, papillae surface area, keratinized layer thickness, and the mitotic index, were determined in 4 papillae per animal using computer-aided light microscope image analysis. For the mitotic index, the number of cells exhibiting mitotic figures was determined using the same microscope as just described, and the final data were expressed as a percentage of 2,000 cells.

\section{Statistical Analysis}

Feedlot performance, carcass characteristics, DMI variation, feeding behavior, selective consumption, and rumen morphometrics measurements were analyzed by a generalized linear model procedure using the statistical software R (version 3.0.3; R Core Team, 2014) according to the following model:

$$
Y_{i j k}=\mu+G_{i}+a_{k(j)}+Y_{j}+G_{i j}+E_{i j k}
$$

where $Y_{\mathrm{ijkl}}=$ dependent variable measured for the ith RFI group and the kth animal within the jth year; $\mu=$ overall mean; $\mathrm{Gi}=$ fixed effect of the ith RFI group, where $\mathrm{i}=$ low, medium, high; $\mathrm{a}_{\mathrm{k}(\mathrm{j})}=$ random effect of the kth animal within the jth year; $Y_{j}=$ fixed effect of the $j$ th year, $j=$ 1,$2 ; \mathrm{GY}_{\mathrm{ij}}=$ interaction term of the ith RFI group within the jth year; and $\mathrm{E}_{\mathrm{ijk}}=$ error term, $\sim \mathrm{N}\left(0, \sigma^{2} \mathrm{e}\right)$.

Tukey's honestly significant difference multiple comparison test was applied for RFI groups. Pearson correlations among performance variables and RFI groups were used, and the most relevant results for discussion are presented in Table 2. Differences were considered significant at a $5 \%$ probability level and a tendency at $10 \%$. 
Table 3. Effect of different residual feed intake (RFI) groups on feedlot performance and carcass characteristics of Nellore yearling bulls consuming high-concentrate diets

\begin{tabular}{|c|c|c|c|c|c|c|c|c|}
\hline \multirow[b]{2}{*}{ Variable } & \multicolumn{3}{|c|}{ RFI group } & \multicolumn{2}{|c|}{ Year } & \multirow[b]{2}{*}{ SEM } & \multicolumn{2}{|c|}{$P$-value } \\
\hline & High & Medium & Low & 1 & 2 & & RFI group & Year \\
\hline$n$ & 25 & 56 & 27 & 48 & 60 & & & \\
\hline \multicolumn{9}{|l|}{ Feedlot performance } \\
\hline RFI, kg/d & $0.888^{\mathrm{a}}$ & $0.042^{b}$ & $-0.920^{\mathrm{c}}$ & -0.006 & 0.012 & 0.145 & $<0.01$ & 0.78 \\
\hline Initial BW, kg & $382.46^{\mathrm{a}}$ & $378.07^{\mathrm{a}}$ & $371.76^{\mathrm{b}}$ & $353.01^{\mathrm{y}}$ & $401.85^{\mathrm{x}}$ & 3.01 & $<0.01$ & $<0.01$ \\
\hline Final BW, kg & 484.04 & 489.48 & 474.74 & $448.26^{\mathrm{y}}$ & $517.24^{\mathrm{x}}$ & 6.72 & 0.25 & $<0.01$ \\
\hline Daily DMI, kg & $10.12^{\mathrm{a}}$ & $9.51^{\mathrm{b}}$ & $8.19^{c}$ & $7.83^{y}$ & $10.72^{x}$ & 0.24 & $<0.01$ & $<0.01$ \\
\hline Daily DMI, \% BW & $2.32^{\mathrm{a}}$ & $2.18^{\mathrm{b}}$ & $1.92^{\mathrm{c}}$ & $1.95^{\mathrm{y}}$ & $2.33^{\mathrm{x}}$ & 0.02 & $<0.01$ & $<0.01$ \\
\hline DMI Variation, \% & $12.28^{\mathrm{b}}$ & $15.14^{\mathrm{a}}$ & $16.24^{\mathrm{a}}$ & $16.70^{x}$ & $12.41^{\mathrm{y}}$ & 0.94 & 0.02 & $<0.01$ \\
\hline DMI Variation, kg & 1.15 & 1.18 & 1.13 & $1.07 \mathrm{y}$ & $1.23^{\mathrm{x}}$ & 0.04 & 0.71 & $<0.01$ \\
\hline $\mathrm{ADG}, \mathrm{kg}$ & 1.14 & 1.25 & 1.16 & $1.02^{\mathrm{y}}$ & $1.34^{\mathrm{x}}$ & 0.04 & 0.20 & $<0.01$ \\
\hline $\mathrm{G}: \mathrm{F}, \mathrm{kg} / \mathrm{kg}$ & $0.112^{\mathrm{b}}$ & $0.131^{\mathrm{a}}$ & $0.143^{\mathrm{a}}$ & 0.131 & 0.126 & 0.004 & $<0.01$ & 0.30 \\
\hline $\mathrm{BW}^{0.75}, \mathrm{~kg}$ & 94.9 & 94.9 & 92.9 & $89.2^{\mathrm{y}}$ & $99.3^{\mathrm{x}}$ & 2.23 & 0.23 & $<0.01$ \\
\hline $\mathrm{DMI} / \mathrm{BW}^{0.75}, \mathrm{~g} / \mathrm{kg}$ & $106.5^{\mathrm{a}}$ & $99.4^{\mathrm{b}}$ & $86.9^{\mathrm{c}}$ & $87.2^{\mathrm{y}}$ & $108.0^{\mathrm{x}}$ & 6.96 & $<0.01$ & $<0.01$ \\
\hline $\mathrm{RG},{ }^{1} \mathrm{~kg} / \mathrm{d}$ & $-0.162^{b}$ & $0.027^{\mathrm{a}}$ & $0.108^{\mathrm{a}}$ & 0.016 & -0.034 & 0.04 & $<0.01$ & 0.19 \\
\hline RIWG, ${ }^{2} \mathrm{~kg} / \mathrm{d}$ & $-0.762^{\mathrm{c}}$ & $-0.066^{b}$ & $0.885^{\mathrm{a}}$ & -0.008 & 0.046 & 0.12 & $<0.01$ & 0.44 \\
\hline Kleiber ratio, g gain $/ \mathrm{kg}$ & 0.012 & 0.013 & 0.012 & 0.011 & 0.014 & 0.002 & 0.27 & $<0.01$ \\
\hline \multicolumn{9}{|l|}{ Carcass characteristics } \\
\hline Initial 12th rib fat, $\mathrm{mm}$ & 2.39 & 2.45 & 2.34 & 2.37 & 2.41 & 0.05 & 0.31 & 0.48 \\
\hline Final 12th rib fat, $\mathrm{mm}$ & $5.57^{\mathrm{a}}$ & $5.25^{\mathrm{a}}$ & $4.71^{\mathrm{b}}$ & $5.49^{\mathrm{x}}$ & $4.86^{\mathrm{y}}$ & 0.09 & $<0.01$ & $<0.01$ \\
\hline 12th rib fat daily gain, $\mathrm{mm}$ & 0.033 & 0.030 & 0.028 & $0.035^{\mathrm{x}}$ & $0.025^{\mathrm{y}}$ & 0.001 & 0.14 & $<0.01$ \\
\hline Initial P8 fat thickness, ${ }^{3} \mathrm{~mm}$ & 2.68 & 2.89 & 2.82 & $2.66^{\mathrm{y}}$ & $2.93^{\mathrm{x}}$ & 0.06 & 0.15 & 0.03 \\
\hline Final P8 fat thickness, mm & $6.31^{\mathrm{a}}$ & $6.17^{\mathrm{a}}$ & $5.43^{\mathrm{b}}$ & $6.15^{\mathrm{x}}$ & $5.78^{\mathrm{y}}$ & 0.10 & $<0.01$ & 0.06 \\
\hline P8 fat daily gain, mm & 0.037 & 0.036 & 0.032 & $0.040^{\mathrm{x}}$ & $0.029^{\mathrm{y}}$ & 0.001 & 0.14 & $<0.01$ \\
\hline $\mathrm{HCW}, \mathrm{kg}$ & 266.77 & 262.66 & 256.07 & $248.77^{y}$ & $274.89^{\mathrm{x}}$ & 3.81 & 0.25 & $<0.01$ \\
\hline Dressing percentage & $55.23^{\mathrm{a}}$ & $53.79^{\mathrm{b}}$ & $54.05^{\mathrm{a}}$ & $55.51^{\mathrm{x}}$ & $53.21^{\mathrm{y}}$ & 0.36 & $<0.01$ & $<0.01$ \\
\hline $\mathrm{KPH}, \mathrm{kg}$ & $3.13^{\mathrm{a}}$ & $2.78^{\mathrm{b}}$ & $2.45^{\mathrm{b}}$ & $3.59^{\mathrm{x}}$ & $1.99^{\mathrm{y}}$ & 0.16 & 0.05 & $<0.01$ \\
\hline $\mathrm{KPH}, \% \mathrm{HCW}$ & 1.19 & 1.08 & 0.96 & $1.43^{\mathrm{x}}$ & $0.72^{\mathrm{y}}$ & 0.05 & 0.07 & $<0.01$ \\
\hline
\end{tabular}

\section{RESULTS}

A single regression equation for DMI was fitted for the study replicated in 2 consecutive years without intercept $(P=0.19)$. Since 12 th rib and P8 fat thickness were not significant in the DMI equation $(P=$ 0.21 and 0.75 , respectively), variables were removed from the model.

The final fitted equation was

DMI $(\mathrm{kg} / \mathrm{d})=2.0163 \times \mathrm{ADG}+0.0934 \times$

$\mathrm{BW}^{0.75}-0.7645 \times$ year, $r^{2}=0.84$.

\section{Pearson Correlation Coefficients}

Pearson correlation was used to assess the correlations among all variables evaluated. However, only the most relevant results will be presented (RFI, DMI, DMI fluctuation, ADG, G:F, ERNDF, RRNDF; Table 2). Year did not have an effect on the correlation values $(P=0.42)$. The correlation between ADG, RRNDF, and RFI was not significant $(P>0.08)$. The RFI was positively correlated with DMI $(P<0.01)$. RFI was negatively correlated with G:F $(P<0.01)$, DMI fluctuation $(P<0.01)$, and ERNDF $(P<0.01)$.

\section{Feedlot Performance and Carcass Characteristics}

Results of the feedlot performance and carcass characteristics are presented in Table 3. The difference in DMI between high- and low-RFI yearling bulls observed in this study was $1.808 \mathrm{~kg}$ DM daily. Low-RFI animals (more efficient) consumed $-0.920 \mathrm{~kg} / \mathrm{d}$, and high-RFI animals (less efficient) consumed $+0.888 \mathrm{~kg} / \mathrm{d}$ for similar ADG. Low-RFI yearling bulls had lower initial BW $(P<$ 
Table 4. Effect of different residual feed intake (RFI) groups on feeding behavior and selective consumption of Nellore yearling bulls consuming high-concentrate diets

\begin{tabular}{|c|c|c|c|c|c|c|c|c|}
\hline \multirow[b]{2}{*}{ Variable } & \multicolumn{3}{|c|}{ RFI group } & \multicolumn{2}{|c|}{ Year } & \multirow[b]{2}{*}{ SEM } & \multicolumn{2}{|c|}{$P$-value } \\
\hline & High & Medium & Low & 1 & 2 & & RFI group & Year \\
\hline$n$ & 25 & 56 & 27 & 48 & 60 & & & \\
\hline \multicolumn{9}{|l|}{ Feeding behavior } \\
\hline Time spent eating, $\min / \mathrm{d}$ & 221.21 & 230.49 & 211.75 & $178.74^{y}$ & $263.56^{\mathrm{x}}$ & 10.16 & 0.36 & $<0.01$ \\
\hline Time spent ruminating, $\min / \mathrm{d}$ & $422.52^{\mathrm{a}}$ & $378.68^{\mathrm{b}}$ & $387.52^{\mathrm{b}}$ & $434.59^{x}$ & $357.88^{\mathrm{y}}$ & 11.55 & $<0.01$ & $<0.01$ \\
\hline Time spent resting, $\min / \mathrm{d}$ & 796.12 & 833.28 & 841.48 & 826.80 & 820.45 & 14.35 & 0.12 & 0.73 \\
\hline Meals per day, no. & 14.99 & 15.02 & 14.75 & 14.39 & 15.44 & 0.80 & 0.97 & 0.27 \\
\hline Daily DMI, ${ }^{1} \mathrm{~kg}$ & $11.21^{\mathrm{a}}$ & $10.79^{\mathrm{a}}$ & $8.87^{\mathrm{b}}$ & $8.66^{y}$ & $11.91^{\mathrm{x}}$ & 0.25 & $<0.01$ & $<0.01$ \\
\hline DMI per meal, $\mathrm{kg}$ & 0.80 & 0.76 & 0.68 & $0.65^{\mathrm{y}}$ & $0.84^{\mathrm{x}}$ & 0.04 & 0.20 & $<0.01$ \\
\hline NDF intake, $\mathrm{kg}$ & $3.29^{\mathrm{a}}$ & $3.35^{\mathrm{a}}$ & $2.74^{\mathrm{b}}$ & 3.09 & 3.17 & 0.10 & $<0.01$ & 0.49 \\
\hline Meal length, min & 15.16 & 15.49 & 15.47 & $12.87^{y}$ & $17.87^{x}$ & 0.73 & 0.94 & $<0.01$ \\
\hline $\mathrm{ERDM},{ }^{2} \mathrm{~min} / \mathrm{kg}$ of $\mathrm{DM}$ & $19.90^{\mathrm{a}}$ & $21.70^{\mathrm{a}}$ & $24.37^{\mathrm{b}}$ & $20.68^{y}$ & $23.30^{\mathrm{x}}$ & 1.07 & 0.03 & 0.05 \\
\hline $\mathrm{RRDM},{ }^{3} \mathrm{~min} / \mathrm{kg}$ of DM & $40.78^{b}$ & $36.36^{\mathrm{a}}$ & $47.25^{\mathrm{c}}$ & $51.61^{x}$ & $31.31^{\mathrm{y}}$ & 0.87 & $<0.01$ & $<0.01$ \\
\hline $\mathrm{ERNDF},{ }^{4} \mathrm{~min} / \mathrm{kg}$ of NDF & $50.39^{\mathrm{a}}$ & $52.23^{\mathrm{a}}$ & $61.03^{\mathrm{b}}$ & $60.84^{x}$ & $48.26^{\mathrm{y}}$ & 2.65 & 0.04 & $<0.01$ \\
\hline RRNDF, ${ }^{5} \mathrm{~min} / \mathrm{kg}$ of NDF & $118.87^{\mathrm{a}}$ & $112.05^{\mathrm{a}}$ & $137.50^{\mathrm{b}}$ & $149.54^{x}$ & $96.07^{y}$ & 4.79 & $<0.01$ & $<0.01$ \\
\hline \multicolumn{9}{|l|}{ Selective consumption ${ }^{6}$} \\
\hline 19-mm screen & $0.98^{\mathrm{b}}$ & $0.97^{\mathrm{b}}$ & $1.24^{\mathrm{a}}$ & 1.10 & 1.03 & 0.006 & 0.09 & 0.42 \\
\hline 8-mm screen & $1.00^{\mathrm{b}}$ & $0.99^{\mathrm{b}}$ & $1.04^{\mathrm{a}}$ & $0.95^{\mathrm{y}}$ & $1.07^{\mathrm{x}}$ & 0.003 & 0.04 & $<0.01$ \\
\hline 1.18-mm screen & 1.00 & 1.00 & 1.00 & $0.98^{\mathrm{y}}$ & $1.02^{\mathrm{x}}$ & 0.007 & 0.34 & $<0.01$ \\
\hline Pan & 1.04 & 1.02 & 1.00 & $1.09^{\mathrm{x}}$ & $0.96^{\mathrm{y}}$ & 0.005 & 0.31 & $<0.01$ \\
\hline
\end{tabular}

${ }^{\mathrm{a}-\mathrm{c}}$ Within a row, for RFI group, means without a common superscript letter $\operatorname{differ}(P<0.05)$.

$\mathrm{x}, \mathrm{y}$ Within a row, for year, means without a common superscript letter differ $(P<0.05)$.

${ }^{1}$ Daily DMI is the DMI of the day that behavior measurements were taken.

${ }^{2} \mathrm{ERDM}=$ eating rate of DM.

${ }^{3} \mathrm{RRDM}=$ rumination rate of DM.

${ }^{4} \mathrm{ERNDF}=$ eating rate of NDF.

${ }^{5} \mathrm{RRNDF}=$ rumination rate of NDF.

${ }^{6}$ Selective consumption $=n$ intake $/ n$ predicted intake, in which $n=$ particle fraction screens of $19 \mathrm{~mm}$ (long), $8 \mathrm{~mm}$ (medium), $1.18 \mathrm{~mm}$ (short), and a pan (fine). Selective consumption values equal to 1 indicate no sorting, those $<1$ indicate selective refusals (sorting against), and those $>1$ indicate preferential consumption (sorting for).

$0.01)$, daily DMI expressed in kilograms $(P<0.01)$ and as a percentage of BW $(P<0.01)$, and DMI based on $\mathrm{BW}^{0.75}(P<0.01)$ than medium- and high-RFI animals. Likewise, low- and medium-RFI yearling bulls presented greater DMI fluctuation expressed in percent $(P=0.04)$, RG $(P<0.01)$, RIWG $(P<0.01)$, and G:F $(P<0.01)$ than high-RFI yearling bulls. However, no significant RFI grouping effect was observed for DMI fluctuation in kilograms $(P=0.71)$, final BW $(P=0.18)$, ADG $(P$ $=0.20), \mathrm{BW}^{0.75}(P=0.23)$, and Kleiber ratio $(P=0.27)$.

No RFI grouping effect was observed for variables of carcass characteristics $(P>0.05)$, except for final 12th rib fat thickness, final P8 fat thickness, dressing percentage, and KPH. Low-RFI yearling bulls had thinner final 12th rib fat $(P<0.01)$ and final P8 fat $(P<0.01)$ than medium- and high-RFI animals. Medium-RFI animals presented decreased dressing percentage when compared to low- and high-RFI animals; high-RFI animals had a larger amount of KPH $(P=0.05)$ than low- and medium-RFI animals.

No significant year effect was observed for RFI $(P=$ 0.78), G:F $(P=0.30), \mathrm{RG}(P=0.19)$, RIWG $(P=0.44)$, and initial 12th rib fat $(P=0.48)$. In year 2 , yearling bulls had greater initial BW $(P<0.01)$, final BW $(P<$ 0.01 ), daily DMI (in kilograms and as a percentage of BW; $P<0.01$ ), DMI variation (in kilograms and as a percentage; $P<0.01)$, ADG $(P<0.01)$, $\mathrm{BW}^{0.75}(P<$ $0.01), \mathrm{DMI} / \mathrm{BW}^{0.75}(P<0.01)$, Kleiber ratio $(P<0.01)$, and initial $\mathrm{P} 8$ fat thickness $(P=0.03)$ and heavier HCW $(P<0.01)$. However, in year 1 , yearling Nellore bulls had greater final 12th rib fat $(P<0.01)$, 12th rib fat daily gain $(P<0.01)$, final P8 fat thickness $(P<0.01)$, P8 fat daily gain $(P<0.01)$, and $\mathrm{KPH}$ (in kilograms and as a percentage of HCW; $P<0.01)$, and increased dressing percentage $(P<0.01)$.

\section{Feeding Behavior and Selective Consumption}

Results related to feeding behavior and selective consumption are presented in Table 4. A significant RFI grouping effect was observed for time spent ruminating $(P<0.01)$, DMI $(P<0.01)$, NDF intake $(P<0.01)$, ERDM $(P=0.03)$, RRDM $(P<0.01)$, ERNDF $(P=$ $0.04)$, and RRNDF $(P<0.01)$. Low-RFI Nellore year- 
Table 5. Effect of different residual feed intake (RFI) groups on rumenitis and rumen morphometrics of Nellore yearling bulls consuming high-concentrate diets

\begin{tabular}{|c|c|c|c|c|c|c|c|c|}
\hline \multirow[b]{2}{*}{ Variable } & \multicolumn{3}{|c|}{ RFI group } & \multicolumn{2}{|c|}{ Year } & \multirow[b]{2}{*}{ SEM } & \multicolumn{2}{|c|}{$P$-value } \\
\hline & High & Medium & Low & 1 & 2 & & RFI group & Year \\
\hline $\begin{array}{ll}n \\
n\end{array}$ & 25 & 56 & 27 & 48 & 60 & & & \\
\hline Rumenitis score & 1.47 & 1.45 & 1.11 & $1.85^{\mathrm{x}}$ & $0.84^{\mathrm{y}}$ & 0.17 & 0.38 & $<0.01$ \\
\hline \multicolumn{9}{|l|}{ Cranial sac } \\
\hline Number of papillae, no. & 45.62 & 43.60 & 43.09 & 43.59 & 44.62 & 2.20 & 0.76 & 0.70 \\
\hline ASA, ${ }^{1} \mathrm{~cm}^{2} / \mathrm{cm}^{2}$ of rumen wall & 25.36 & 26.26 & 22.69 & $21.86^{\mathrm{y}}$ & $27.69^{x}$ & 1.47 & 0.15 & $<0.01$ \\
\hline Papillae area, $\mathrm{cm}^{2}$ & $0.55^{\mathrm{a}, \mathrm{b}}$ & $0.60^{\mathrm{a}}$ & $0.50^{\mathrm{b}}$ & $0.49^{y}$ & $0.61^{\mathrm{x}}$ & 0.02 & 0.07 & $<0.01$ \\
\hline \multicolumn{9}{|l|}{ Ventral sac } \\
\hline Papillae height, mm & 4.35 & 4.40 & 4.04 & $2.94^{\mathrm{y}}$ & $5.32^{\mathrm{x}}$ & 0.16 & 0.34 & $<0.01$ \\
\hline Papillae width, mm & 0.41 & 0.39 & 0.37 & $0.35^{\mathrm{y}}$ & $0.43^{\mathrm{x}}$ & 0.01 & 0.12 & $<0.01$ \\
\hline Papillae surface area, $\mathrm{mm}^{2}$ & 1.78 & 1.81 & 1.54 & $1.13^{\mathrm{y}}$ & $2.29^{\mathrm{x}}$ & 0.11 & 0.22 & $<0.01$ \\
\hline Keratinized layer thickness, $\mu \mathrm{m}$ & 20.43 & 20.36 & 19.31 & 20.09 & 19.96 & 0.36 & 0.12 & 0.62 \\
\hline Mitotic index, $\%$ basal cells & 7.47 & 7.08 & 6.77 & $11.32^{\mathrm{x}}$ & $2.89^{y}$ & 0.55 & 0.78 & $<0.01$ \\
\hline
\end{tabular}

ling bulls had lower DMI and NDF intake, and they took longer to consume and ruminate a kilogram of DM and a kilogram of NDF when compared to medium- and high-RFI animals. Also, Nellore yearling bulls presenting low- and medium-RFI spent less time ruminating than high-RFI animals $(P<0.01)$. No significant $(P>$ $0.10)$ RFI effect was observed for selective consumption (Table 4) when diet and orts samples were sieved on a $1.18-\mathrm{mm}$ screen and pan. Nevertheless, low-RFI Nellore yearling bulls consumed more particles larger than $19 \mathrm{~mm}(P=0.09)$ and $8 \mathrm{~mm}(P=0.04)$ than medium- and high-RFI animals.

No significant year effect was observed for time spent resting $(P=0.73)$, meals per day $(P=0.27)$, NDF intake $(P=0.49)$, and selection of particles larger than 19 $\mathrm{mm}(P=0.42)$. Yearling bulls, in year 2 , spent more time eating $(P<0.01)$ and less time ruminating $(P<0.01)$, had greater daily DMI $(P<0.01)$ and DMI per meal $(P<$ $0.01)$, sorted for particles larger than $8 \mathrm{~mm}(P<0.01)$ and $1.18 \mathrm{~mm}(P<0.01)$, and had longer meal length $(P<$ $0.01)$. Also, yearling Nellore bulls in year 2 sorted against particles smaller than $1.18 \mathrm{~mm}$ and presented slower intake of a kilogram of DM $(P<0.01)$, faster intake of a kilogram of NDF $(P<0.01)$, and faster rumination of a kilogram of both DM $(P<0.01)$ and NDF $(P<0.01)$.

\section{Rumenitis and Rumen Morphometrics}

Results for rumenitis score and rumen morphometrics are presented in Table 5. No significant $(P>0.10)$ RFI grouping effect was observed for rumenitis score $(P=0.38)$, NOP $(P=0.76)$, ASA $(P=0.15)$, papillae height $(P=0.34)$, papillae width $(P=0.12)$, keratinized layer thickness $(P=0.12)$, papillae surface area $(P=$
$0.22)$, or mitotic index $(P=0.78)$. However, Nellore yearling bulls classified as low RFI tended to have smaller MPA than medium-RFI animals $(P=0.07)$.

No significant year effect was observed for number of papillae $(P=0.70)$ and keratinized layer thickness $(P=0.62)$. However, yearling bulls in year 2 had greater ASA $(P<0.01)$, papillae height $(P<0.01)$, and papillae width $(P<0.01)$; larger MPA $(P<0.01)$ and papillae surface area $(P<0.01)$; and lower rumenitis score $(P<0.01)$ and mitotic index $(P<0.01)$.

\section{DISCUSSION}

\section{RFI Group}

The variables final BW and ADG were not affected by different RFI groups, which confirms that RFI in cattle is phenotypically independent of growth and body size, as expected. Also, the correlation between ADG and RFI was not significant, as reported by Nascimento et al. (2015). Likewise, the data obtained in this study corroborate those found by Herd and Bishop (2000), Arthur et al. (2001), and Basarab et al. (2003), who have reported positive correlations between RFI and DMI and negative correlation with G:F in Hereford, Charolais, Limousin, and Gelbvieh cattle. Sobrinho et al. (2011) also reported a reduction of the daily DMI, expressed as percentage of BW, in low-RFI Nellore cattle.

Low-RFI Nellore yearling bulls presented a reduction in $\mathrm{DMI}$ based on $\mathrm{BW}^{0.75}$ and greater RG and RIWG and showed that low-RFI animals are more efficient in feed utilization than high-RFI animals, as also observed by Nascimento et al. (2015) with Nellore cattle. LowRFI Nellore yearling bulls presented greater G:F than 
high-RFI animals because the reduced DMI and had similar ADG. The greater preference of low-RFI animals for particles larger than 19 and $8 \mathrm{~mm}$ may explain the fact that these animals took longer to consume and ruminate a kilogram of both NDF and DM, which may have led to greater ruminal retention time, potentially explaining the lower DMI (Davis et al., 2014). On the basis of this fact, several studies have shown that lowRFI animals have greater nutrient digestibility (Herd et al., 2004; Nkrumah et al., 2006; Cruz et al., 2010) than high-RFI animals, which may also explain the greater G:F of low-RFI yearling bulls. Also, the lower NDF intake presented by low-RFI animals may have contributed to the greater selection of particles larger than $8 \mathrm{~mm}$ to maintain proper rumen function. Moreover, because low-RFI animals take longer to consume and ruminate a kilogram of DM and NDF, daily DMI may have become less constant (negative correlation) and, consequently, increased DMI variation overall. On the basis of this fact, a smaller amount of substrates may have been available for rumen fermentation and VFA production in low-RFI animals, especially propionate, which is responsible for promoting the growth of metabolically active ruminal papillae (Costa et al., 2008). In this study, low-RFI yearling bulls tended to have smaller papillae area than medium-RFI animals; however, different RFI groups did not affect the ASA in this study. In addition, animals with low-RFI may have spent less energy to produce methane (Nkrumah et al., 2006) on the basis of the lower NDF intake, and considering the poor quality of the NDF in the diets provided by the sugarcane bagasse, low-RFI yearling Nellore bulls may have saved some energy that contributed to the similar ADG and HCW across the different RFI groups in this study.

Galyean et al. (1992) reported that DMI fluctuation above $10 \%$ reduced ADG by $6 \%$ and $\mathrm{G}$ :F by $7 \%$, basically because of the negative effect of subclinical acidosis; however, ruminal $\mathrm{pH}$ was not measured in that study, and this concept often has been applied by the cattle feeding industry. Nevertheless, several trials (Soto-Navarro et al., 2000; Schwartzkopf-Genswein et al., 2004; Bevans et al., 2005; Schwartzkopf-Genswein et al., 2011) have not observed a negative effect of greater DMI variation on feedlot performance. Schwartzkopf-Genswein et al. (2011) evaluated the performance of animals with low, medium, and high G:F and concluded that animals with better G:F showed the greatest DMI fluctuation. In the present study, greater DMI fluctuation was observed in the more efficient animals (low- and medium-RFI animals), and it did not negatively impact the incidence of rumenitis or the rumen wall absorptive surface area. Low-RFI animals had lower NDF intake, and to offset the smaller amount of fiber to stimulate rumination and maintain rumen function, they consumed more particles larger than 19 and $8 \mathrm{~mm}$. This fact may have contributed to the greater DMI fluctuation in low-RFI animals since larger particles stay longer in the rumen.

Regarding feeding behavior, no differences were observed for time spent eating, and the values for this variable were similar to the ones observed by Bingham et al. (2009), who utilized Brangus heifers classified according to RFI. Nkrumah et al. (2006) reported shorter meal length and lower DMI per meal in low-RFI animals when compared to high-RFI bulls. In the present study, no RFI grouping effect was observed for meal length $(P>0.10)$, which suggests that the reduced daily DMI presented by low-RFI animals may be partially explained by ERDM, RRDM, ERNDF, and RRNDF. Herd et al. (2004) reported that high-RFI animals spent $13 \%$ more time to capture and masticate the diet than low-RFI animals, with increased energy waste for such activity. Animals grouped as low- and medium-RFI spent significantly $(P<0.01)$ less time ruminating than the high-RFI animals, which also may have contributed to improved G:F in these animals as the net energy for maintenance may have been reduced.

With respect to the carcass characteristics, low-RFI animals had thinner final 12th rib and final P8 fat and a smaller amount of KPH than high-RFI animals. On the basis of the fact that net energy for maintenance may have been reduced, low-RFI animals had lower DMI, which may have delayed the beginning of carcass fat deposition in these animals and contributed to improving G:F as low-RFI animals became more efficient. Richardson et al. (2001), Basarab et al. (2003), Arthur and Herd (2008), Lancaster et al. (2009), and Santana et al. (2012) also observed a reduction in back fat deposition of Bos taurus breeds and Nellore bulls grouped as low RFI. Nevertheless, as in this study, other studies also reported low and nonsignificant correlations between RFI and carcass characteristics (Baker et al., 2006; Barwick et al., 2009).

\section{Year}

Yearling bulls from year 2 showed greater ADG because of greater DMI (both in kilograms and as a percentage of BW), as no differences were observed across the years for G:F. On the basis of this fact, the DMI variation was greater for animals from year 2 because of the greater DMI. Yearling bulls from year 1 may have presented lower DMI because of the greater sorting against particles larger than 8 and $1.18 \mathrm{~mm}$, which may have compromised proper rumen function for increasing lactic acid and VFA production (Owens et al., 1998). In addition, cattle from year 1 took longer to consume and ruminate a kilogram of NDF, and as a result, those animals presented greater rumenitis scores and smaller 
ASA, which may partially explain the poorer performance when compared to animals from year 2. Yearling bulls from year 2 animals had larger papillae and, consequently, larger ASA because of greater DMI, resulting in decreased mitotic index, which is indicative of VFA absorption and epithelium adaptation as propionate is most responsible for most cellular multiplication of metabolically active papillae (Costa et al., 2008). Furthermore, animals from year 1 were lighter at the beginning of the trial, which also may explain in part the poorer performance of those animals. According to NRC (2000), lighter animals (year $1=353.01 \mathrm{~kg}$ ) require more $\mathrm{MP}$ and less $\mathrm{NE}_{\mathrm{g}}$ than heavier animals (year 2: $401.85 \mathrm{~kg}$ ), and as the diets provided were the same in both years, yearling Nellore bulls from year 1 may have accelerated fat deposition as they had greater final 12th rib fat and final P8 fat thickness, greater KPH (in kilograms and as a percentage of $\mathrm{HCW}$ ), and increased dressing percentage than animals from year 2. However, as yearling bulls from year 2 presented greater final BW, they also had heavier HCW when compared to animals from year 1 .

\section{Conclusion}

Low-RFI Nellore yearling bulls sorted for particles larger than 19 and $8 \mathrm{~mm}$, which partially explains the lower DMI presented for those animals. Even presenting lower DMI, in general, low-RFI animals had a similar performance when compared to both medium- and highRFI yearling bulls without negatively impacting the incidence of rumenitis and the rumen wall absorptive surface area. The concept that greater DMI fluctuation has been associated with poorer feedlot performance needs to be further investigated. Also, low-RFI Nellore yearling bulls had a significant decrease in carcass fat deposition, which might be detrimental to meat quality, so genetic selection based solely on this criterion is not advised.

\section{LITERATURE CITED}

AOAC. 1990. Official methods of analysis. 15th ed. Assoc. Off. Anal. Chem., Arlington, VA.

AOAC. 1995. Official methods of analysis. 16th ed. AOAC Int., Gaithersburg, MD.

AOAC. 1997. Official methods of analysis. 16th ed. AOAC Int., Arlington, VA.

Archer, J. A., P. F. Arthur, R. M. Herd, P. F. Parnell, and W. S. Pitchford. 1997. Optimum postweaning test for measurement of growth rate, feed intake, and feed efficiency in British breed cattle. J. Anim. Sci. 75:2024-2032.

Arthur, J. P. F., and R. M. Herd. 2008. Residual feed intake in beef cattle. R. Bras. Zootec. 37 81(E. Suppl.):269-279. doi:10.1590/ S1516-35982008001300031
Arthur, P. F., J. A. Archer, D. J. Johnson, R. M. Herd, E. C. Richardson, and P. F. Parnell. 2001. Genetics and phenotypic variance and covariance components for feed intake, feed efficiency and other postweaning traits in Angus cattle. J. Anim. Sci. 79:2805-2811. doi:/2001.79112805x

Baker, S. D., J. I. Szasz, T. A. Klein, P. S. Kuber, C. W. Hunt, J. B. Glaze Jr., D. Falk, R. Richard, J. C. Miller, R. A. Battaglia, and R. A. Hill. 2006. Residual feed intake of purebred Angus steers: Effects on meat quality and palatability. J. Anim. Sci. 84:938945. doi:/2006.844938x

Barwick, S. A., M. L. Wolcott, D. J. Johnston, H. M. Burrow, and M. T. Sullivan. 2009. Genetics of steer daily and residual feed intake in two tropical beef genotypes, and relationships among intake, body composition, growth and other post-weaning measures. Anim. Prod. Sci. 49:351-366. doi:10.1071/EA08249

Basarab, J. A., M. A. Price, J. L. Aalhus, E. K. Okine, W. M. Snelling, and K. L. Lyle. 2003. Residual feed intake and body composition in young growing cattle. Can. J. Anim. Sci. 83:189-204. doi:10.4141/A02-065

Berry, D. P., and J. J. Crowley. 2012. Residual intake and body weight gain: A new measure of efficiency in growing cattle. J. Anim. Sci. 90:109-115. doi:10.2527/jas.2011-4245

Bevans, D. W., K. A. Beauchemin, K. S. Schwartzkopf-Genswein, J. J. McKinnon, and T. A. McAllister. 2005. Effect of rapid or gradual grain adaptation on subacute acidosis and feed intake by feedlot cattle. J. Anim. Sci. 83:1116-1132. doi:/2005.8351116x

Bigham, M. L., and W. R. McManus. 1975. Whole wheat grain feeding of lambs: Effects of roughage and wheat grain mixtures. Aust. J. Agric. Res. 26:1053-1062.

Bingham, G. M., T. H. Friend, P. A. Lancaster, and G. E. Carstens. 2009. Relationship between feeding behavior and residual feed intake in growing Brangus heifers. J. Anim. Sci. 87:2685-2689. doi:10.2527/jas.2009-1851

Costa, S. F., M. N. Pereira, L. Q. Melo, J. C. Resende Júnior, and M. L. Chaves. 2008. Alterações morfológicas induzidas por butirato, propionato e lactato sobre a mucosa ruminal e a epiderme de bezerros - I Aspectos histológicos. Arq. Bras. Med. Vet. Zootec. 60:1-9. doi:10.1590/S0102-09352008000100001

Crowley, J. J., M. McGee, D. A. Kenny, D. H. Crews Jr., R. D. Evans, and D. P. Berry. 2010. Phenotypic and genetic parameters for different measures of feed efficiency in different breeds of Irish performance-tested beef bulls. J. Anim. Sci. 88:885-894. doi:10.2527/jas.2009-1852

Cruz, G. D., J. A. Rodríguez-Sánchez, J. W. Oltjen, and R. D. Sainz. 2010. Performance, residual feed intake, digestibility, carcass traits, and profitability of Angus-Hereford steers housed in individual or group pens. J. Anim. Sci. 88:324-329. doi:10.2527/ jas.2009-1932

Davis, M. P., H. C. Freetly, L. A. Kuehn, and J. E. Wells. 2014. Influence of dry matter intake, dry matter digestibility, and feeding behavior on body weight gain of beef steers. J. Anim. Sci. 92:3018-3025. doi:10.2527/jas.2013-6518

Fox, D. G., L. O. Tedeschi, T. P. Tylutki, J. B. Russell, M. E. Van Amburgh, L. E. Chase, A. N. Pell, and T. R. Overton. 2004. The Cornell net carbohydrate and protein system model for evaluating herd nutrition and nutrient excretion. Anim. Feed Sci. Technol. 112:29-78. doi:10.1016/j.anifeedsci.2003.10.006

Galyean, M. L., K. J. Malcolm, and G. C. Duff. 1992. Performance of feedlot steers fed diets containing laidlomycin propionate or monensin plus tylosin, and effects of laidlomycin propionate concentration on intake patterns and ruminal fermentation in beef steers during adaptation to a high-concentrate diet. J. Anim. Sci. 70:2950-2958. 
Heinrichs, J., and P. Kononoff. 1996. Evaluating particle size of forages and TMRs using the Penn State Particle Size Separator. Dairy and Animal Science, Wallingford. p. 1-14.

Herd, R. M., and S. C. Bishop. 2000. Genetic variation in residual feed intake and its association with other production traits in British Hereford cattle. Livest. Prod. Sci. 63:111-119. doi:10.1016/ S0301-6226(99)00122-0

Herd, R. M., V. H. Oddy, and E. C. Richardson. 2004. Biological basis for variation in residual feed intake in beef cattle. I. Review of potential mechanisms. Aust. J. Exp. Agric. 44:423-430. doi:10.1071/EA02220

Kleiber, M. 1947. Body size and metabolic rate. Physiol. Rev. 27:511-541.

Lancaster, P. A., G. E. Carstens, F. R. B. Ribeiro, L. O. Tedeschi, and D. H. Crews. 2009. Characterization of feed efficiency traits and relationships with feeding behavior and ultrasound carcass traits in growing bulls. J. Anim. Sci. 87:1528-1539. doi:10.2527/ jas.2008-1352

Ministério da Ciência, Tecnologia e Inovação Conselho Nacional de Controle de Experimentação Animal. 2013. Diretriz brasileira para o cuidado e a utilização de animais para fins científicos e didáticos. http://www.mct.gov.br/upd_blob/0234/234054.pdf. (Accessed 21 June 2015).

Nascimento, C. F., R. H. Branco, S. F. M. Bonilha, J. N. S. G. Cyrillo, J. A. Negrão, and M. E. Z. Mercadante. 2015. Residual feed intake and blood variables in young Nellore cattle. J. Anim. Sci. 93:1318-1326. doi:10.2527/jas.2014-8368

Nkrumah, J. D., E. K. Okine, G. W. Mathinson, S. Schmid, C. Li, J. A. Basarab, M. A. Price, Z. Wang, and S. S. Moore. 2006. Relationships of feedlot, feed efficiency, performance, and feeding behavior with metabolic rate, methane production, and energy partitioning in beef cattle. J. Anim. Sci. 84:145-153. doi:/2006.841145x

NRC. 2000. Nutrient requirements of beef cattle. 7th rev. ed. Natl. Acad. Press, Washington, DC.

Odongo, N. E., O. AlZahal, M. I. Lindinger, T. F. Duffield, E. V. Valdes, S. P. Terrel, B. W. McBride. 2006. Effects of mild heat stress and grain challenge on acid-base balance and rumen tissue histology in lambs. J. Anim. Sci. 84:447-455. doi:/2006.842447x

Oliveira, C. A., and D. D. Millen. 2014. Survey of the nutritional recommendations and management practices adopted by feedlot cattle nutritionists in Brazil. Anim. Feed Sci. Technol. 197:64 75. doi:10.1016/j.anifeedsci.2014.08.010

Owens, F. N., D. S. Secrist, W. J. Hill, and D. R. Gill. 1998. Acidosis in cattle: A review. J. Anim. Sci. 76:275-286.

Pacheco, R. D. L., D. D. Millen, N. DiLorenzo, C. L. Martins, C. T. Marino, M. V. Fossa, S. L. Beier, A. DiCostanzo, P. H. M. Rodrigues, and M. D. B. Arrigoni. 2012. Effects of feeding a multivalent polyclonal antibody preparation on feedlot performance, carcass characteristics, rumenitis, and blood gas profile in Bos indicus biotype yearling bulls. J. Anim. Sci. 90:18981909. doi:10.2527/jas.2010-3521

Perkins, T. L., R. D. Green, and K. E. Hamlin. 1992. Evaluation of ultrasonic estimates of carcass fat thickness and longissimus muscle area in beef cattle. J. Anim. Sci. 70:1002-1010.

R Core Team. 2014. R: A language and environment for statistical computing. R Found. Stat. Comput., Vienna.
Resende Júnior, J. C., L. S. Alonso, M. N. Pereira, M. G. R. Magallanes, M. G. Duboc, E. C. Oliveira, and L. Q. Melo. 2006. Effect of the feeding pattern on rumen wall morphology of cows and sheep. Braz. J. Vet. Res. Anim. Sci. 43:526-536.

Richardson, E. C., and R. M. Herd. 2004. Biological basis for variation in residual feed intake in beef cattle. 2. Synthesis of results following divergent selection. Aust. J. Exp. Agric. 44:431-440. doi:10.1071/EA02221

Richardson, E. C., R. M. Herd, V. H. Oddy, J. M. Thompson, J. A. Archer, and P. F. Arthur. 2001. Body composition and implications for heat production of Angus steers progeny of parents selected for and against residual feed intake. Aust. J. Exp. Agric. 41:1065-1072. doi:10.1071/EA00095

Robles, V., L. A. González, A. Ferret, X. Manteca, and S. Calsamiglia. 2007. Effects of feeding frequency on intake, ruminal fermentation, and feeding behavior in heifers fed high-concentrate diets. J. Anim. Sci. 85:2538-2547. doi:10.2527/jas.2006-739

Santana, M. H. A., P. Rossi Jr., R. Almeida, and D. C. Cucco. 2012. Feed efficiency and its correlations with carcass traits measured by ultrasound in Nellore bulls. Livest. Sci. 145:252-257. doi:10.1016/j.livsci.2012.02.012

Schutt, K. M., P. F. Arthur, and H. M. Burrow. 2009. Brahman and Brahman crossbred cattle grown on pasture and in feedlots in subtropical and temperate Australia. 3. Feed efficiency and feeding behavior of feedlot finished animals. Anim. Prod. Sci. 49:452-460. doi:10.1071/EA08083

Schwartzkopf-Genswein, K. S., K. A. Beauchemin, T. A. Mcallister, D. J. Gibb, and M. Streeter. 2004. Effect of feed delivery fluctuations and feeding time on ruminal acidosis, growth performance, and feeding behavior of feedlot cattle. J. Anim. Sci. 82:33573365. doi:/2003.8114_suppl_2E149x

Schwartzkopf-Genswein, K. S., D. D. Hickman, M. A. Shah, C. R. Krehbiel, B. M. A. Genswein, R. Silasi, D. G. Gibb, D. H. Crews, and T. A. McAllister. 2011. Relationship between feeding behavior and performance of feedlot steers fed barley-based diets. J. Anim. Sci. 89:1180-1192. doi:10.2527/jas.2010-3007

Simas, J. M. C., A. V. Pires, I. Susin, F. A. P. Santos, C. Q. Mendes, R. C. Oliveira Jr., and J. J. R. Fernandes. 2008. Efeitos de fontes e formas de processamento do amido na utilização de nutrientes e parâmetros ruminais de vacas em lactação. Arq. Bras. Med. Vet. Zootec. 60(5):1128-1134. doi:10.1590/S010209352008000500014

Sobrinho, T. L., R. H. Branco, S. F. M. Bonilha, A. M. Castilhos, L. A. Figueiredo, A. G. Razook, and M. E. Z. Mercadante. 2011. Residual feed intake and relationships with performance of Nellore cattle selected for post weaning weight. Rev. Bras. Zootec. 40(4):929-937. doi:10.1590/S1516-35982011000400030

Soto-Navarro, S. A., C. R. Krehbiel, G. C. Duff, M. L. Galyean, M. S. Brown, and R. L. Steiner. 2000. Influence of feed intake fluctuation and frequency of feeding on nutrient digestion, digesta kinetics, and ruminal fermentation profiles in limit-fed steers. J. Anim. Sci. 78:2215-2222. doi:10.2527/2000.7882215x

Turner, J. W. 1980. Genetic and biological aspects of zebu adaptability. J. Anim. Sci. 50:1201-1205.

Van Soest, P. J., J. B. Robertson, and B. A. Lewis. 1991. Symposium: Carbohydrate methodology, metabolism, and nutritional implications in dairy cattle. Methods for dietary fiber, neutral detergent fiber, and nonstarch polysaccharides in relation to animal nutrition. J. Dairy Sci. 74:3583-3597. doi:10.3168/jds.S00220302(91)78551-2 